\title{
Learning Dynamics Analysis Based on Computer-Human Interaction
}

\author{
http://dx.doi.org/10.3991/ijet.v8i5.3028 \\ YANG Sheng ${ }^{1}$, GUO Lei ${ }^{1}$, YU Wensen ${ }^{1}$, LIU Changyong ${ }^{1}$, MENG Shimin ${ }^{2}$ \\ ${ }^{1}$ Wuyi University, Wuyishan, Fujian, China \\ ${ }^{2}$ Orient Potential Software Technology Co.,Ltd, BCSM, Wuyishan, Fujian, China,
}

\begin{abstract}
Unlike doctors, teachers are not able to carry out personality analysis and teaching diagnosis via using various tools and data. So how can we build the exact teaching environment, collect the data of study process, and perform the evidence-based teaching? To answer the question, computers are introduced to observe study activities, which will surely become an important trend just as in other fields. By designing the cognition process oriented novel study observation software system based on Computer-Human Interaction (CHI) to realize the online observation of study process, massive amount of information that can hardly be obtained by traditional education and psychology is able to be efficiently collected. According to dynamics, data of study process was analyzed to obtain the variables of cognitive dynamics such as study speed, acceleration, average speed, key points, learning kinetic energy and so on. All these parameters reflected the personalities of the learners, which will provide useful information for teaching, training and diagnosis.
\end{abstract}

Index Terms-CHI; Agent; Cognitive Structure, Manifold; Category; Evidence-based teaching

\section{INTRODUCTION}

Mechanics and dynamic system method are important paradigms to investigate the objective physical world and to reveal the core regulations of things development. From Newton's laws to quantum mechanics, the "seemed" orderly physical rules were discovered; from chaos theory to complex network, the "seemed" out-of-order complicated regulations were revealed in the physical world. However, it is just a start to use mechanics and dynamic system method for the brain and psychological world investigation. For instance, the book of cognition science and dynamic theory, in which the research clue of cognitive science and dynamics was brought up, was published by T.van Gelder and R.Port in 1995. It was considered the declaration of the third competitive paradigm in cognitive science ${ }^{[1-2]}$. The dynamic comprehensive way of cognition was provided in the publication done by G.G.Globus, S.S.Robertson, E.Thelen and L.B.Smith ${ }^{[3-4]}$.

Mechanics and dynamic system cognitive paradigm has been trying to describe regulation of the varied mental cognition with time. In 1995, T.van Gelder held an opinion in his dynamics hypothesis that the cognitive system in the cognitive world was a kind of dynamic system, and it would be the best to comprehend the cognitive system in the view of dynamics. The dynamics hypothesis describes the cognition based on mathematics and dynamic system theory, where the internal cognitive process of the cognitive subject interacted with environment is explained using the mathematic concepts like state space, attractor, track, certainty, chaos, etc. Differential equations are used to describe the cognitive trace of cognitive subject in cognitive space. In other words, cognition is described as the multi-dimensional space constituted by all possible thoughts and behaviors of the cognitive subject, and especially, it is thoroughly investigated via the thought track of the cognitive subject under certain internal pressure and certain environment. The thoughts and behaviors of the cognitive subject are controlled by differential equations. The variables of the system are evolving continuously, and the system obeys the nonlinear differential equations which are often complex and certain.

At present, the cognitive systems include: 1) cyclical motor behavior model: S.S Robertson roughly described the circulation in the spontaneous actions and behaviors of new-born babies using dynamics; 2) olfactory bulb model: the article "How brains make chaos in order to make sense of the world" published by C. A. Skarda and W. J. Freeman in 1987 has roughly drawn the outline of this model and experiments have been carried out to a certain extent. This is a delicate model that was brought up based on the investigation of olfactory neurological processes, and assisted by the complex dynamic system theory to describe various complex states of the nervous system within sensory organs, including the description of chaotic neuron activities and its regular track; 3) motivational oscillatory theory: the most important issue in motivational oscillatory theory is how to choose the right parameters for the system. Since the system is extremely sensitive to the initial values, "Changing one parameter of dynamic system is changing its whole dynamics."

The dynamic system theory has provided the timedependant naturalistic explanation for the continuous cognitive behaviors, which is not able to be explained by other paradigms. Generally, other paradigms neglect the concept of time. But the information exchange between humans' brains and the environment happens all the time, and it is changing continuously, so the transient continuous cognition varies with time.

The dominance of dynamic system theory is that it can multivariately describe cognition. And it is a theory that can be empirically tested, can analyze and correct the differential equations that describe cognitive systems and also can use existing techniques to solve all these equations.

Now the science of learning becomes the advanced thermal point of cognition science ${ }^{[6]}$, the evidence-based teaching is the new thought of education development. It 
promotes the development of high quality, high efficient education method, brings up new concepts like Precision Teaching (PT), etc., ${ }^{[7-8]}$ and teachers are expected to carry out teaching activities referring to more data and tools like doctors. Doubtless, it is the work of great value to construct the learning dynamics model.

Similar to other dynamics models, the key point to build a learning dynamics model is data collection. How to collect data of study and what kinds of tools to be used for observation and collection become the vital questions. In natural science like physics, observation is closely related to the tools, and there is no doubt that the computer is the most widely used tool. So can we use computers as the tool for monitoring learning activities? That is, to observe learning process and collect learning data via $\mathrm{CHI}$. Unlike the data collection methods in general psychological and behavioral science, the cognitive coupling constituted by $\mathrm{CHI}$ Agent and brain can collect massive amount data via online interaction ${ }^{[9-11]}$.

This paper demonstrates how we can design the CHI learning environment to collect data of online mental arithmetic training, and perform the preliminary mechanical analysis of massive information to understand the cognitive system in the view of dynamics.

\section{OBSERVATION FRAME OF CHI LEARNING MANIFOLD}

From observation, the learning information collection space is the interface of Computer-Human Interaction scenario. Facing the complex CHI learning situation, how should we collect the learning process? Observation is closely related to reconfiguration, namely, the hypnosis of the observation subject existence for the manifold is implied when designing the method for observation.

Definition 1. Cognitive manifold: Manifold is the space that is locally of Euclidean space features. When the learning process happens in the cognitive manifold, Euclidean spaces are the GHI cognitive situations. Considering the manifold theory, the Euclidean space is a metric space which is able to be observed. Likewise, we can observe the CHI learning situations to realize the local measurement of cognitive manifold, and hence to study the whole or major property of cognitive manifold.

Definition 2. BSM: Brain, $\mathrm{CHI}$ interfaces and software system are contained in CHI study system, which can be divided by three parts: brain cognitive subject - situation of coupling - manifold information, or "BSM" for short.

Definition 3. Cognitive frame: In the manifold theory, the local Euclidean space can be measured by coordinate or frame. We can set the cognitive frame to measure the cognitive scenario. The frame structure reflects our understanding of cognitive situations, and the cognitive frame determines which parts of the information in cognitive situations we should collect and which are not. According to the cognitive background, we believe that the cognitive content, cognitive operation and cognitive mathematics are those we need to collect, that is, we build cognitive content, operation and mathematical frame to collect and measure the cognitive condition, which is called COM frame ${ }^{[11]}$.

Definition 4. Cognitive symbol sequence: Supposing that we used CHI method to do dictation for students, 1) the computer pronounced [buk], 2) students were required to input words in the textbox, 3) the computer then judged the answers to be right or wrong. For most study software, if a student typed $\mathrm{b}$, and then $\mathrm{u}, \mathrm{k}, \mathrm{o}$, o on the keyboard, they probably only recorded the result as true or false for the dictation, which was represented by 0,1 . In contrast, based on the COM frame, it is required to record the operation process, the amount of time cost, etc for finishing the word dictation. Record like a) "b-bu-buk-bok-book" was kept with the dictation time (2013-5-1 14:00) and the duration (10s) instead of b) b-u-k-o-o or c) using just 0,1 to simply show the result. We consider method (a) the cognitive process oriented information collecting way ${ }^{[12]}$, or the COM based observation mode of cognition.

Morphism, category and category theory can be regarded as the important way for imaging the happening of humans' cognitive tools. It provides new perspectives to make people clearly see the dynamic variations of the pathway from concreteness to formality during the cognitive process. Y. J. Pi, a famous psychologist, started the category based cognitive research in as early as $1960 \mathrm{~s}^{[13]}$. It is believed there is category morphism existing between brain cognitive system and computer system, so their relationship can be described via category theory.

Definition 5. BSM category system: For ComputerHuman Interaction, assuming brain cognitive subject was Category B, situation of coupling was Category S, manifold information was Category $M$, and they altogether were named Category BSM or Computer-Human Coupling Category. The following morphisms exist in the Category BSM:

1) Cognitive morphism: The morphism exists between the brain cognitive subjects. Based on brain thinking, it is the correlation of the cognitive spaces inside brain.

2) Symbolic morphism: The morphism exists in external manifold information. It is the morphism of symbol, logic and operation system based on symbolic calculation of computers.

3) Coupling morphism: It is founded between cognitive morphism and symbolic morphism in coupling situation to realize the two way link between them.

Theorem 1. Cognitive Natural Isomorphism of Information - Cognitive Space: morphism $\boldsymbol{f} \in \operatorname{hom}(\mathrm{X}, \mathrm{Y})$ is equal to $(f: X \approx Y)$. If the two sided inverse of $f, f$ ${ }^{1}$ hom $(\mathrm{Y}, \mathrm{X})$, does exist, namely, equivalent morphism exists between information - cognitive category, the cognitive category subjects are equivalent, which is called the Cognitive Natural Isomorphism Theorem, as is shown in Figure 1.

$$
\begin{aligned}
& \operatorname{hom}(B, S) \times \operatorname{hom}(S, M) \rightarrow \operatorname{hom}(B, M) \\
& \operatorname{hom}(M, S) \times \operatorname{hom}(S, B) \rightarrow \operatorname{hom}(M, B)
\end{aligned}
$$

Theorem 2. Cognitive Coupling Transformation of Information - Cognitive Space: According to category functor principle, the cognitive functor keeps the coupling transformation among BSM, and maintains the covariance of cognitive regulation between inner cognitive morphism and manifold information morphism, which is shown in Figure 2.

The Cognitive Natural Isomorphism Theorem and the Cognitive Coupling Transformation Theorem have maintained the uniformity of cognitive subjects and the covariance of cognitive regulations for cognitive-information spaces. It is the mathematical foundation of BSM cognitive observation and imaging. 


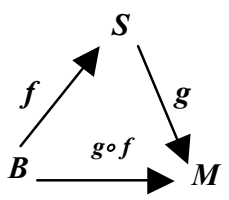

Fugure 1. Cognitive natural isomorphism

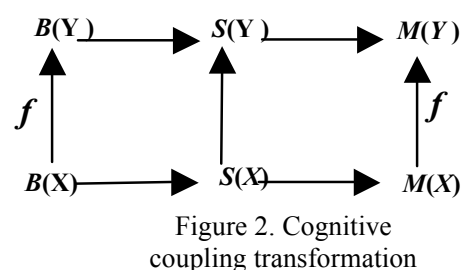

coupling transformation
By referring to the category theory, we have obtained The Cognitive Natural Isomorphism Theorem and the Cognitive Coupling Transformation Theorem[11], which provide mathematical foundation for the $\mathrm{CHI}$ learning observation. Based on the morphism theory, the data collected during $\mathrm{CHI}$ learning process reflect the motion state and learning process of the cognitive space subjects.

\section{BSM BASED LEARNING DYNAMICS ANALYSIS}

Unlike the traditional psychology and education observation at present, the CHI study observation can collect data continuously, precisely and massively. Similarly, the astronomical radio telescope can perform continuous observation of the space, which is a significant progress comparing to the manual observation. When facing massive amounts of data, how can we perform dynamics modeling and analysis?

We define the fundamental elements of learning dynamic system as learning objects. The contents of learning objects basically agree with the concept of objects in computer science. Now we think we should use the CHI based learning objects observation to observe the properties of learning objects and learning process, instead of the traditional manual ways or questionnaires.

1) Basic objects of learning dynamic system: dots and lines are the fundamental elements of $\psi$ network. In the visual cognitive network, the dot represents the cognitive object $\boldsymbol{P}$, and the properties of learning dots include basic ones like name, size, color, etc. Line $\boldsymbol{L}$ stands for the relationship among dots $\boldsymbol{P}$, with the properties of name, size, color, direction, etc. Dots and lines are the fundamental topology elements of study space. $\boldsymbol{L}$ and $\boldsymbol{P}$ can constitute geometric graphs and network visual objects, and hence can form the dynamic structure of learning activities.

2) Learning process variables: times of training $\mathbf{N}$, duration of training $\mathbf{T}$ and result of each training $\mathbf{R}$.

3) Learning dynamics process: description of time span and learning dynamics process.

4) Learning dynamics variables: learning process speed, learning process acceleration, key points.

5) Learning personality analysis: according to the features of learning process, the learners' personalities analysis including types and learning abilities are given.

\section{OBSERVATIONAL EXPERIMENT OF MENTAL ARITHMETIC LEARNING PROCESS}

The CHI software system is designed based on specific learning process to perform the learning process observation. Generally, the observation procedures are listed as follows:

1) Interfaces of mental arithmetic CHI learning scenarios: the learning scenario for $\mathrm{CHI}$ is the learning observation target, and the learning process will be finished in this interface. Considering the requirement, $\mathrm{CHI}$ zones are deployed in the learning scenario to collect the operation information.

For example, when we design the mental arithmetic learning system, the left one is questions and keyboard is located on the right, meanwhile, the rain drops are continuously falling down. Before they hit the ground, the answer to this question should be given by using the digital keyboard and pressing the "Enter" button to provide the first solution. If the number inside the textbox is not the result shown in "rain drops" in the left scenario, it will be counted as false for one time, and the "rain drops" still exist and keep dropping. Otherwise, it will be counted a success and "rain drops" will disappear automatically. The time remaining and scores are shown on the right corner.

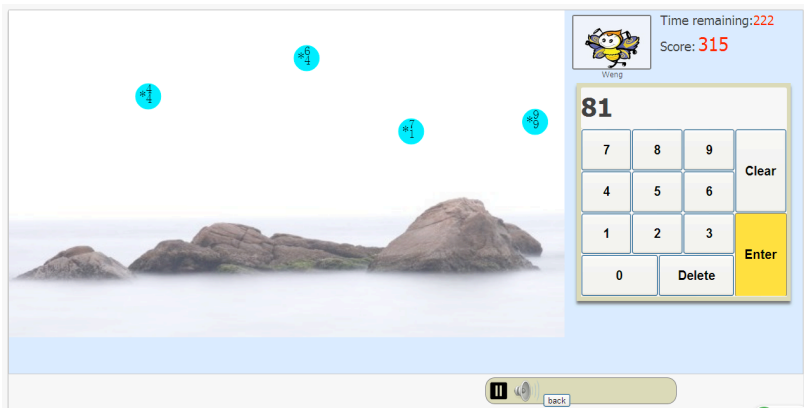

Fugure 3. CHI interface of mental arithmetic training information collection

2) Mental arithmetic training information collection: collecting process information based on the contents of learning. 10 points will be gained for a correct answer and 5 points will be deducted for a wrong one. The overall score will be calculated by 5 minutes as a training unit.

3) Mental arithmetic multi-dimensional variables analysis: collection creation is the foundation of mathematic research, and also the vital step for cognitive data processing. Based on individuals, groups, genders, locations, etc., the collection of multi-dimensional learning objects is built; based on the learning time and duration, learners' ages, grades and areas, the multi-scale learning collection can be created.

4) Dynamic analysis of mental arithmetic learning: The training times are set as the $\mathrm{x}$ axis, and the score of 5 minute training result is the y axis to create the coordinate description of mental arithmetic training. It is called "the progress curve of mental arithmetic", similar to the physical speed in mechanical system.

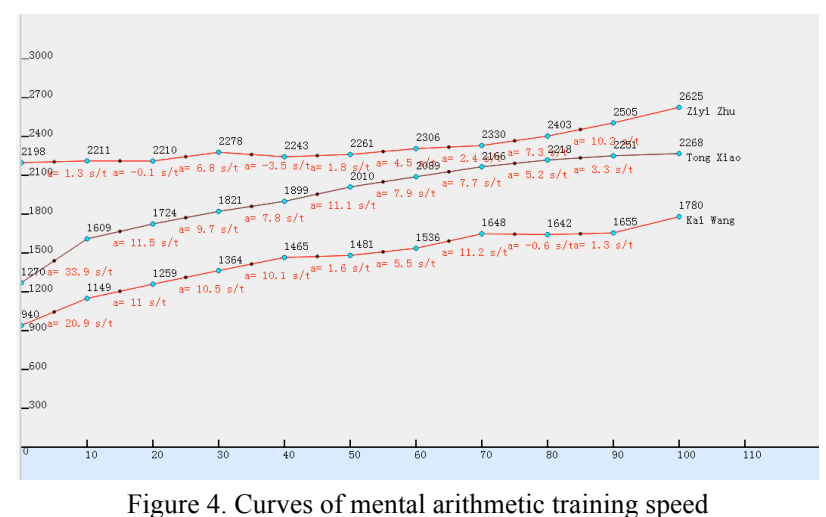

We can calculate the average speed within certain times according to the formula below, where $\mathrm{Y}$ is the obtain 
score, $\mathrm{m}, \mathrm{n}$ are the times on $\mathrm{X}$ axis and $\mathrm{V}$ is the average mental arithmetic speed.

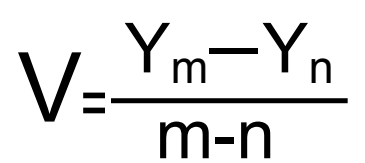

To precisely understand the status of students' mental arithmetic learning progress, we create a concept just as the acceleration in physics.

$$
a=\frac{V_{m}-V_{n}}{m-n}
$$

In this equation, a represents the improving acceleration, $\mathrm{Y}$ stands for the training time of $\mathrm{m}, \mathrm{n}$ on $\mathrm{X}$ axis. According to the above formula, the learning acceleration of the three students shown in Figure 4 can be described in the figure below, where $m-n=1$.

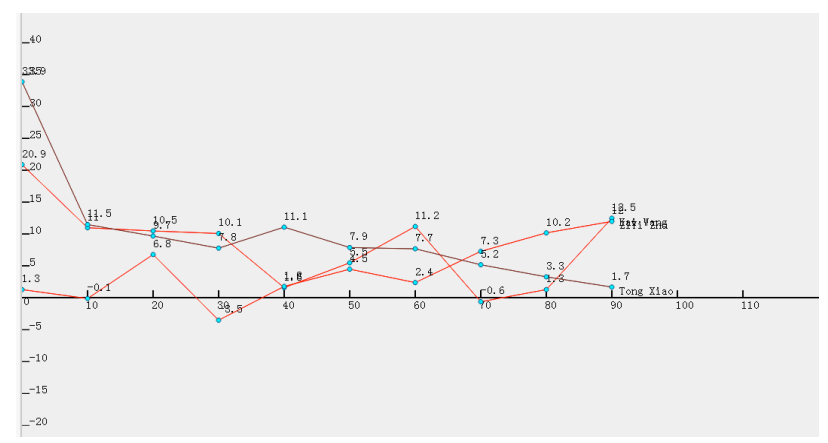

Figure 5. Curves of mental arithmetic training acceleration

When analyzing the acceleration curves, we find that all curves have their minimum points, representing that the improvement of students is the slowest at these points or even go back. The time to reach the minimum point and the value of the lowest acceleration $\left(a_{\min }\right)$ show the student's personality. The times of training performed to reach $\mathrm{a}_{\min }$ are represented by $\mathrm{Ta}_{\min }$. Similar to traditional psychological research, the phenomenon like multiincrease or negative growth could possibly happen to students. For instance, Figure 4 shows $a_{\min }$ and corresponding $\mathrm{Ta}_{\min }$ of three students:

$$
\begin{aligned}
\mathrm{a}_{\min } & =-3.5,-0.6,+2.4 \\
\mathrm{Ta}_{\min } & =40, \quad 80, \quad 100
\end{aligned}
$$

According to the acceleration curves, most students show the $U$ type trend of improvement, which also accords well with the psychological principles. We can divide the whole training period into three parts: $U$ type downward, $U$ type bottom and $U$ type upward. Through the figure, we can clearly notice that the speed of the training score improvement shows a monotonically decreasing trend within certain times, less than 40 times, for example; however, after certain times, it will show a monotonic increase. For instance, after 70 times, the scores of most students show an upward trend. This can provide very important reference for teaching.

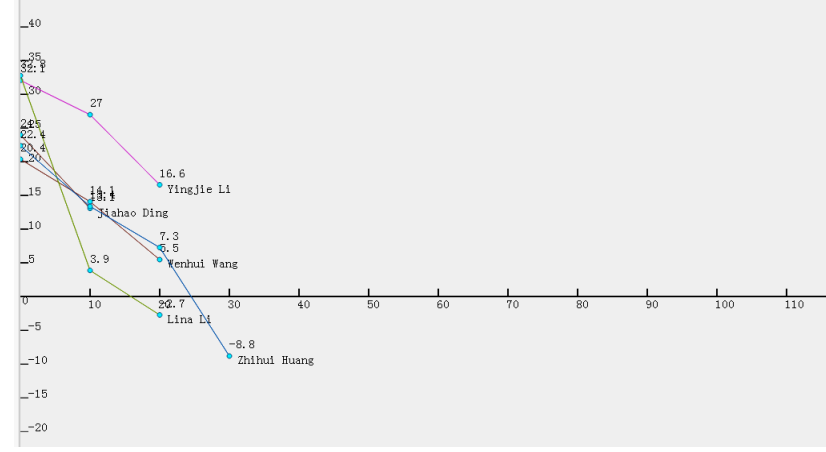

Figure 6. The monotonically decreasing part of mental arithmetic training

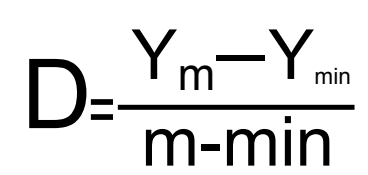

Here we define $Y_{0}$ as the enhancement of the $U$ type minimum part, $\mathrm{Ym}$ is the enhancement of the initial point, $\mathrm{D}$ stands for the average acceleration when reaching the lowest enhancement region, and its value represents the time and acceleration for students to reach the improving limitation, indicating the personalities of these students.

For example, we have calculated some students' D value to be $0.71,1.53,1.32$, which show the improvement personalities of students in the $U$ type downward trend region.

Based on the fundamentals of psychology, the noneimproving region of mental arithmetic training is also the very important information, that is, when students just stop improving after certain times, which is different from the above $\mathrm{D}$ value. What we need to investigate are the points where students just stop improving, or zero improving point $\mathrm{Z}$, instead of those backward points.
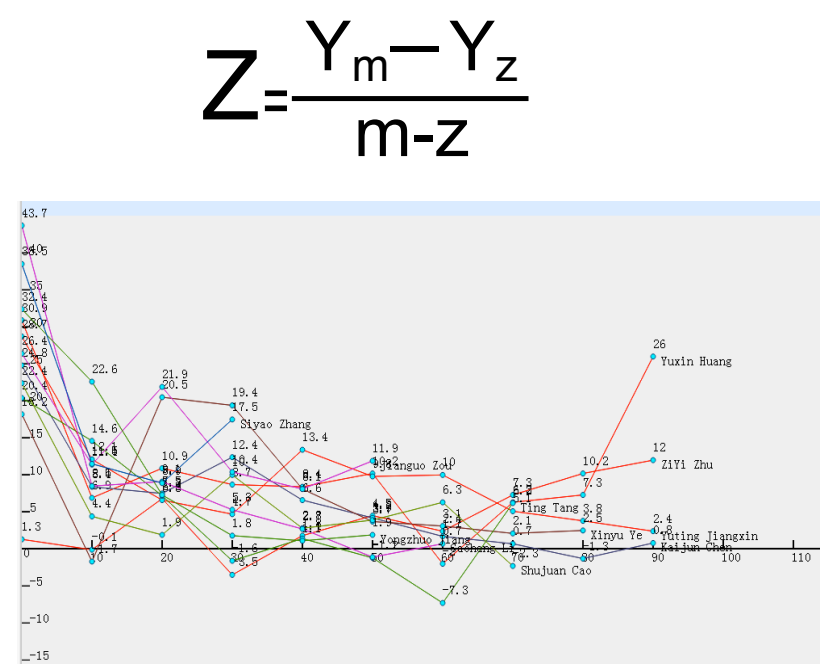

Figure 7. U type of mental arithmetic training

According to the data analysis, we find that after around 20, 30 and 40 times of training, the scores of students have reached 900, 1149 and 1200 points, respectively, and their personalities are not quite different between each other. However, after 1200 points, they begin to show different 
personalities. We call these points where differences are hard to distinguish "common personality points" and in contrast, "different personality points" for others. For example, after 50 times of practice, there are differences existing among their scores. Teachers can utilize these points to perform their teaching activities in educational practice.

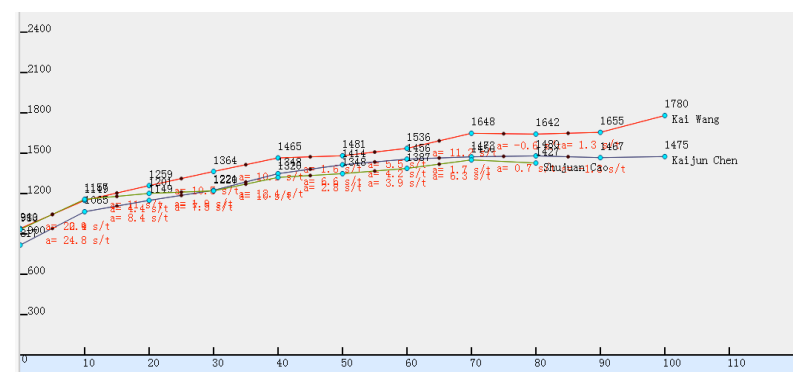

Figure 8 . The common personality points and different personality points in curves of mental arithmetic training acceleration

\section{ACKNOWLEDGMENT}

The Computer-Human Interaction environment is the combination of information and psychology world. By designing a proper $\mathrm{CHI}$ software system, information collection of learning process can be realized by computers and massive amount of data can be obtained.

We have demonstrated the Cognitive Natural Isomorphism Theorem and the Cognitive Natural Transformation Theorem based on category theory, guaranteeing the uniformity of cognition-information space subjects and the covariance of processes.

According to the mechanical frame of cognition and learning, we have designed the mental arithmetic CHI system to collect data of learning process, and carried out mechanics-oriented data analysis. Hence the data including learning speed, acceleration, etc are obtained, describing the dynamic process of learning.

Based on dynamic analysis, we preliminarily find the "different personality and common personality" points, which provides important reference to teaching training and diagnosis.

\section{ACKNOWLEDGMENT}

The paper is supported by the following funds: the Education Ministry Fund in Fujian Province (JA12321), The Special Fund for Industry Development and Scientific Research in Nanping, Fujian (N2011WZ04) and the Key Scientific Project Fund in Nanping, Fujian (N2012Z06-7).

\section{REFERENCES}

[1] Robert Frederick Port,Timothy Van Gelder.Mind As Motion: Explorations in the Dynamics of Cognition. MIT Press, 1995.

[2] Van Gelder,Tim J. "Dynamic approaches to cognition."The MIT Encyclopedia of cognitive sciences (1999): 244-246.

[3] Robert M. French,Elizabeth Thomas.The Dynamical Hypothesis in Cognitive Science: A Review Essay of Mind As Motion[J] ,2001.

[4] ESTHER THELEN and LINDA B. SMITH. Dynamic Systems Theories. Handbook of Child Psychology . 2006:258-312.

[5] Skarda C A, Freeman W J. How brains make chaos in order to make sense of the world [J]. Behavioral and brain sciences, 1987, 10(2): 161-195. http://dx.doi.org/10.1017/S0140525X00047336

[6] National Science Foundation. Converging Technologies for Improving Human Performance [M],2003 Kluwer Academic Publishers (currently Springer): 215-265. http://www.wtec.org/ConvergingTechnologies/Report/NBIC repo rt.pdf, http://en.wikipedia.org/wiki/Converging Technologies for Improving_Human_Performance.

[7] Moran,D.J., Malott,R.W., Yan Xiao, Ran Shao. Evidence based education method. Chinese Light Industry Press, 2006, 9.

[8] Tim Harford. Revolution of Evidence Based Education http://www.ftchinese.com/story/001050259 [EB/OL].

[9] Guangyou Xu, Linmi Tao, Dapeng Zhang, et al. The dual relation between physical space and information space. Chinese Science Bulleti, V51(5), 2006, 610-616.

[10] MENG Simon, CHENG Rengui. Cognitive Coupling States Based on Tree Cognitive Fields, 2011 International Conference on Computer Communication and Management Proc. of CSIT vol.5 (2011) C (2011) IACSIT Press, Singapore,pp593597.

[11] MENG Shimin, LIU Changyong, et al. Cognitive Digital Manifold Based on CHI. Computer and Information technology. 2013.1:18,49 .

[12] LIU Changyong, MENG Shimin. Information Sequence of Cognitive-oriented 1:1 Digital Classroom. Journal of Changchun Teachers College (Natural Science), December, 2012, Vol. 31(12):121124.

[13] J. Piyajie, et al. Morphism and category: comparison and transformation. Shanghai, East China Normal University Press, September, 2005, 11-37.

\section{AUTHORS}

YANG Sheng male, born in 1966, he has a Master's degree and is now an assistant professor. His research fields include Man-machine Interaction, Artificial Intelligence, Data Mining and Intelligent Algorithms. (e-mail: yangsheng@wuyiu.edu.cn).

MENG Shimin was with UlinkM.com INC. He is now with the Department of Brain Cognitive Structure Mapping Lab (e-mail: mengshimin@ulinkm.com).

Submitted 19 June 2013. Published as re-submitted by the authors 13 October 2013. 